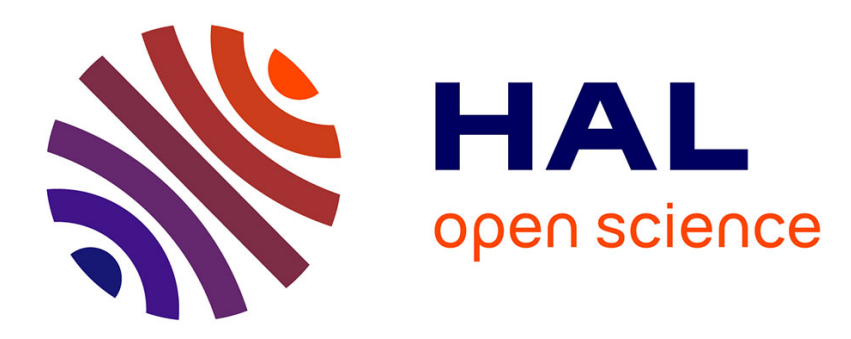

\title{
A probabilistic rate theory connecting kinetics to thermodynamics
}

Denis Michel

\section{To cite this version:}

Denis Michel. A probabilistic rate theory connecting kinetics to thermodynamics. 2017. hal$01569569 \mathrm{v} 7$

\author{
HAL Id: hal-01569569 \\ https://hal.science/hal-01569569v7
}

Preprint submitted on 15 Mar 2018

HAL is a multi-disciplinary open access archive for the deposit and dissemination of scientific research documents, whether they are published or not. The documents may come from teaching and research institutions in France or abroad, or from public or private research centers.
L'archive ouverte pluridisciplinaire HAL, est destinée au dépôt et à la diffusion de documents scientifiques de niveau recherche, publiés ou non, émanant des établissements d'enseignement et de recherche français ou étrangers, des laboratoires publics ou privés. 


\title{
A probabilistic rate theory connecting kinetics to thermodynamics*
}

\author{
Denis Michel ${ }^{\ddagger}$
}

¥ Universite de Rennes1-IRSET. Campus de Villejean. 35000 Rennes France. Email: denis.michel@live.fr.

\begin{abstract}
Kinetics and thermodynamics are largely disconnected in current theories because Arrhenius activation energies $\left(E_{a}\right)$ have strictly no influence on equilibrium distributions. A first step towards the incorporation of rate theories in thermodynamics is the identification of the pre-exponential term of the Arrhenius equation as an entropic quantity. A second step examined here is the possible contribution of $E_{a}$ in equilibrium landscapes. Interestingly, this possibility exists if envisioning the energetic exponential term of Arrhenius rate constants as the probability that the energy of the reactant is sufficient for the transition. This radically new approach encompasses Maxwell-Boltzmann distributions and solves inconsistencies in previous theories, in particular on the role of temperature in kinetics and thermodynamics. These probabilistic rate constants are then reintroduced in dynamic systems to provide them with the two distinct facets of time: the time step and the time arrow.
\end{abstract}

Keywords: Kinetics; equilibrium; heat capacity; enthalpy; entropy; Gibbs free energy.

Notes to readers: The unitless statistical entropy usually written $H$ will be renamed here $\mathcal{S}$ to avoid confusion with enthalpy. The letter $C$ will be used for heat capacity but not for concentration.

\section{Introduction}

Rate constants are the drivers of all dynamic systems and are widely used in physical chemistry and modeling studies. By the restrictions they impose on chemical transitions, rate constants prevent our organized world from falling directly into its state of weaker free energy and maximal entropy. Yet the profound nature of these constants remains obscure and the main focus of this study is to clarify their energetic component. After multiple attempts of description, its most common expression remains the exponential factor of Arrhenius [1, 2]. In its classical version (without tunneling effects), the empirical Arrhenius equation

$$
k=A \mathrm{e}^{-\frac{E_{a}}{k_{B} T}}
$$

where $k_{B}$ is the Boltzmann constant and $T$ is the temperature, includes (i) a so-called preexponential factor $A$, containing the elementary frequency and configurational restrictions, and (ii) a unitless exponential function containing an energy barrier called activation energy $E_{a}$. In the present paper, the exponential factor of Arrhenius will be reformulated as the probability to reach a threshold energy, as initially proposed but incompletely appraised in $[3$. This simple hypothesis will lead to major changes in thermodynamic relationships.

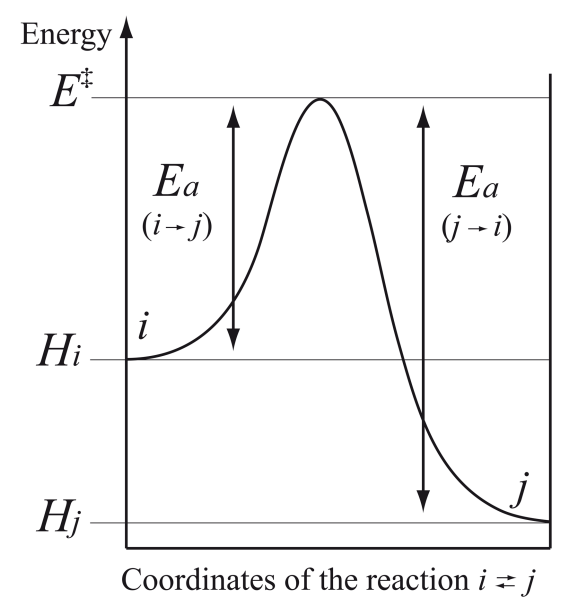

Figure 1. Representation of the energy barrier according to the Arrhenius principle. The barrier is supposed to restrict reaction kinetics but to have no influence on equilibrium ratios. $E^{\ddagger}$ is a fixed energy threshold necessary for crossing the barrier in both directions and $H$ are the mean enthalpies of reaction of the particles.

Using the notations of Fig.1, the Arrhenius approach

\footnotetext{
*Reference: Michel, D. 2018. A probabilistic rate theory connecting kinetics to thermodynamics. Physica A 503, 26-44
} 
reads

$$
k_{i j}=A_{i j} \mathrm{e}^{-\left(E^{\ddagger}-H_{i}\right) / k_{B} T}
$$

and for the reverse reaction

$$
k_{j i}=A_{j i} \mathrm{e}^{-\left(E^{\ddagger}-H_{j}\right) / k_{B} T}
$$

In equilibrium the forward and reverse fluxes equalize such that

$$
n_{i}^{e q} k_{i j}=n_{j}^{e q} k_{j i}
$$

giving

$$
\begin{aligned}
\left(\frac{n_{i}}{n_{j}}\right)_{e q} & =\frac{k_{j i}}{k_{i j}}=K_{j i}=\frac{A_{j i}}{A_{i j}} \mathrm{e}^{\left(E^{\ddagger}-H_{i}-E^{\ddagger}+H_{j}\right) / k_{B} T} \\
& =\frac{A_{j i}}{A_{i j}} \mathrm{e}^{-\left(H_{i}-H_{j}\right) / k_{B} T}=\frac{A_{j i}}{A_{i j}} \mathrm{e}^{-\Delta H / k_{B} T}
\end{aligned}
$$

$E^{\ddagger}$ disappears. The equilibrium ratio is independent of the energetic barrier and depends only on the difference of energy between the reactants. These currently admitted relationships can be naively questioned.

\section{Some concerns and alternative proposal}

\subsection{On the universality of $k_{B} T$ as a mean energy}

$1 / k_{B} T$ is introduced in statistical mechanics as a constant called $\beta$ corresponding to a Lagrangian multiplier or a distribution parameter, and finally connected to temperature by analogy with classical thermodynamics. This introduction of $k_{B} T$ is elegant and perfectly rational in this context but could be too restrictive for an extension to more complex systems. $k_{B} T$ is concretely useful here to adimension the exponent of the Arrhenius exponential and is usually interpreted as the mean particle energy. This interpretation holds in the kinetic theory of ideal gases but is much less clear for complex chemical systems in which the mean reactional energies are precisely different for each type of particle.

\subsection{Insensitivity of dynamic equilibria to activation energies}

The accepted conclusion that the presence and the height of energy barriers do not participate to equilibrium ratios between the reactants, can appear not completely intuitive because one could expect that increasing the height of the barrier exponentially amplifies the differential capacity of the reactants to jump over, including in equilibrium. By contrast, in the potential surface of the transition state theory 4, the activated complexes reside on mountain crests but their energy levels do not influence the populational distributions.

\subsection{A non-probabilistic exponential law}

The exponential term of the Arrhenius law has certain appearences of a probability, but as explained in the following section, as it stands it cannot correspond to the exponential distribution. The purpose of the present study is precisely to examine the theoretical consequences of the hypothesis that it is a probability of sufficient energy for the transition. This simple hypothesis justified later leads to the novel formulas

$$
P(\text { sufficient energy })=\mathrm{e}^{-\frac{E^{\ddagger}}{H}}=\mathrm{e}^{-\left(1+\frac{E a}{H}\right)}
$$

which gives the equilibrium relationship

$$
\begin{aligned}
\ln \left(\frac{n_{i}}{n_{j}}\right)_{e q} & =\ln \frac{A_{j i}}{A_{i j}}+\left(1-\frac{H_{i}}{H_{j}}\right) \frac{E^{\ddagger}}{H_{i}} \\
& =\ln \frac{A_{j i}}{A_{i j}}+\left(1-\frac{H_{i}}{H_{j}}\right)\left(1+\frac{E_{a}}{H_{i}}\right)
\end{aligned}
$$

whose structure is completely different from the usual result

$$
\ln \left(\frac{n_{i}}{n_{j}}\right)_{e q}=\ln \frac{A_{j i}}{A_{i j}}+\frac{H_{j}-H_{i}}{k_{B} T}=\frac{G_{j}-G_{i}}{k_{B} T}
$$

In Eq.(4), the pre-exponential terms $A$ disappear from the latter form containing Gibbs free energies $G$. This modification results from the identification of the pre-exponential factor as an entropic parameter, which can then be incorporated in the Gibbs free energies. This pertinent operation will also be used here and applied to Eq. (3b), but let us first explain the entropic meaning of the pre-exponential factor. This factor can be defined as $A=\tau^{-1} \Omega^{-1}$ where $\tau^{-1}$ is the universal thermal frequency (Section 6.2.2), giving their time ${ }^{-1}$ unit to the rate constants, and $\Omega^{-1}$ is the reciprocal of the number of possible configurations of the reactant, one of which allowing the reaction to proceed, as simply illustrated in Fig.2. As a consequence, $\ln \left(A_{j i} / A_{i j}\right)$ can be identified, after elimination of the identical frequencies, as the difference of statistical entropies

$$
\ln \frac{A_{j i}}{A_{i j}}=\ln \frac{\Omega_{i j}}{\Omega_{j i}}=\mathcal{S}_{i j}-\mathcal{S}_{j i}
$$

Note the inversion of the suffixes between the ratios of $A$ and $\Omega$. In addition, if a given reactant is involved in several reactions, one can assume that it is not necessary to precise the nature of the product in reactional entropy, because it is expected to be the same for all the reactions starting from this reactant. Indeed, even if each reaction requires a specific reactional configuration, 
this configuration belongs the same set of total configurations $\Omega$ of this reactant. Converting configurational into thermodynamic entropy through the Boltzmann formula $\mathcal{S}=S / k_{B}$, allows to detail Eq.(4) step-by-step

$$
\begin{aligned}
\ln \left(\frac{n_{i}}{n_{j}}\right)_{e q} & =\ln \frac{A_{j}}{A_{i}}+\frac{H_{j}-H_{i}}{k_{B} T} \\
& =\ln \frac{\Omega_{i}}{\Omega_{j}}+\frac{H_{j}-H_{i}}{k_{B} T} \\
& =\frac{S_{i}-S_{j}}{k_{B}}+\frac{H_{j}-H_{i}}{k_{B} T} \\
& =\frac{H_{j}-H_{i}-T\left(S_{j}-S_{i}\right)}{k_{B} T} \\
& =\frac{\left(H_{j}-T S_{j}\right)-\left(H_{i}-T S_{i}\right)}{k_{B} T} \\
& =\frac{G_{j}-G_{i}}{k_{B} T}
\end{aligned}
$$

Such a fusion of entropy and enthalpy into free energy will be retained here for whole systems but not for individual reactants, because molecular entropy and enthalpy play different roles in reactions, which are described by different probabilities (Fig.2).

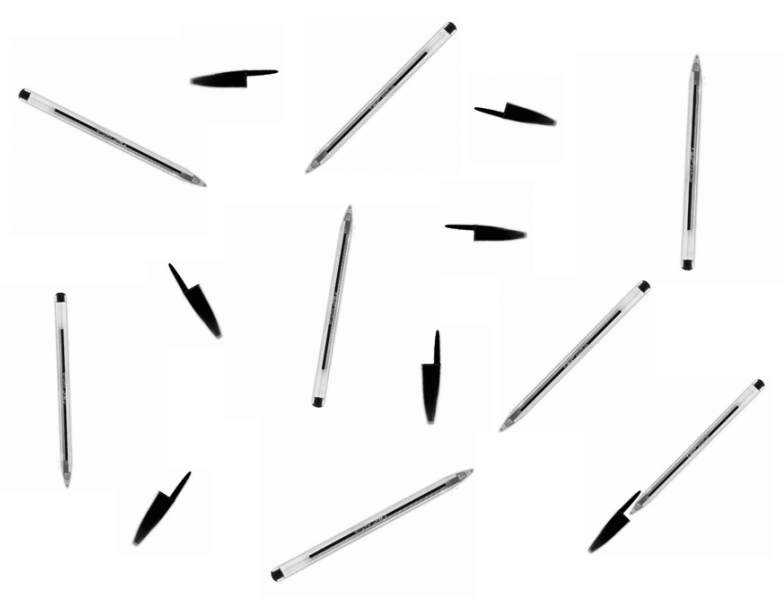

Figure 2. Randomly moving pens and caps simply illustrate the concept of reactional entropy. The set of their equiprobable relative arrangements (which would be much larger in $3 \mathrm{D})$ is noted $\Omega$, out of which only one, of probability $1 / \Omega$, allows the assembly of the caps and the pens, as shown at the bottom right. But even when conveniently positioned, the pen and the cap can stably associate only if the energy of their collision is sufficient. This second condition is quantified from 0 to 1 in the energetic term of rate constants defined here. The configurations illustrated in this figure with separate components, can also be conceived for internal domains of the same molecule.

Eq.(3b) contrasts with Eq.(4) in that it introduces a role for activation energies in equilibrium distributions.
Large $E_{a}$ do not invert but accentuate the differences determined in both cases by the relative energy levels. This point results from a mathematical interpretation of the exponential factor.

\section{A new probabilistic interpreta- tion of the exponential factor}

\subsection{The exponential distribution}

The exponential component of the Arrhenius law and of the Boltzmann distribution, can be simultaneously derived from the exponential distribution, which is basically mathematical. According to this law, the probability that a particle from an homogeneous population with an average number of energy quanta per particle $\langle\mathcal{E}\rangle$, reaches a fixed threshold number $\mathcal{E}^{\ddagger}$, is

$$
P\left(\mathcal{E} \geqslant \mathcal{E}^{\ddagger}\right)=\mathrm{e}^{-\frac{\mathcal{E}^{\ddagger}}{\langle\mathcal{E}\rangle}}
$$

Since $\mathcal{E}^{\ddagger}$ is a temperature-independent threshold and $\langle\mathcal{E}\rangle$ is temperature dependent, temperature remains at the denominator of the exponent, but an important difference with current rate equations is the absence of $k_{B} T$. $k_{B} T$ is generally regarded as the mean single particle energy, acceptable for purely kinetic gases, but not for all heterogeneous systems containing different kinds of energies. An other problem even more critical with the Arrhenius equation of Eq.(1), is that in absence of energetic barrier $\left(E_{a}=0\right)$, the exponential is equal to 1 , suggesting that reactions are no longer energetically restricted but only affected by the pre-exponential factor. In other words, the mean particle energy $k_{B} T$ would be fully sufficient for all the transitions. This result is clearly inconsistent with the view of $k_{B} T$ as a mean energy because of a basic principle: the average particle energy in a homogeneous population is no way reached by all the particles. Indeed, even when $E_{a}=0$, that is to say when the barrier corresponds to the mean particle energy, not all the particles have a sufficient energy. In the new interpretation, $E_{a}=0$ (equivalent to $E^{\ddagger}=H$ in Fig. 1 and to $\left.\mathcal{E}^{\ddagger}=\langle\mathcal{E}\rangle\right)$ predicts a probability of $1 / \mathrm{e}(0.37)$. Even more strangely when setting $H=0$, the new formula gives an exponential of zero, which means that the transition is impossible; but the traditional Arrhenius exponential is non-zero, temperature-dependent and the same for any energyless reactant $\left(\operatorname{Exp}\left[-E^{\ddagger} / k_{B} T\right]\right)$, which makes little sense.

\subsection{The rule for particle interconversion}

The present approach also fundamentally differs from the classical one in equilibrium. The relative populations of interconvertible species are determined not only by the 
difference of their mean energies, but also by the barriers. Interconversion between particles of different nature $i$ and $j$ is possible when either a particle $i$ or a particle $j$, has accumulated a minimal number of energy quanta, denoted below by $\mathcal{E}_{i, j}^{\ddagger}$, which is the same for the conversions $i \rightarrow j$ and $j \rightarrow i$. For a particle of type $i$, the probability of this accumulation is simply given by the exponential distribution $P\left(X_{i} \geqslant \mathcal{E}_{i, j}^{\ddagger}\right)=\mathrm{e}^{-\mathcal{E}_{i, j}^{\ddagger} /\left\langle\mathcal{E}_{i}\right\rangle}$ 3]. As the same holds for the particles of type $j$, when $\left\langle\mathcal{E}_{i}\right\rangle>\left\langle\mathcal{E}_{j}\right\rangle$, the particles of type $i$ would switch more probably than the reverse. As a consequence, the system spontaneously evolves until the reciprocal fluxes equalize. The numbers of particles in the sub-populations $i$ and $j$ necessarily adjust such that

$$
n_{i}^{e q} A_{i j} \mathrm{e}^{-\mathcal{E}_{i, j}^{\ddagger} /\left\langle\mathcal{E}_{i}\right\rangle}=n_{j}^{e q} A_{j i} \mathrm{e}^{-\mathcal{E}_{i, j}^{\ddagger} /\left\langle\mathcal{E}_{j}\right\rangle}
$$

or

$$
\left(\frac{n_{i}}{n_{j}}\right)_{e q}=\frac{A_{j i}}{A_{i j}} \mathrm{e}^{\mathcal{E}_{i, j}^{\ddagger}\left(\frac{1}{\left\langle\mathcal{E}_{i}\right\rangle}-\frac{1}{\left\langle\mathcal{E}_{j}\right\rangle}\right)}
$$

Returning to the correspondence between the unitless pre-exponential term and entropy, this equation takes the form

$$
\ln \left(\frac{n_{i}}{n_{j}}\right)_{e q}=\overbrace{\mathcal{S}_{i}-\mathcal{S}_{j}}^{\text {Entropic term }}+\overbrace{\mathcal{E}_{i, j}^{\ddagger}\left(\frac{1}{\left\langle\mathcal{E}_{i}\right\rangle}-\frac{1}{\left\langle\mathcal{E}_{j}\right\rangle}\right)}^{\text {Energetic term }}
$$

Eq.(7) can also be written with the activation energy $\mathcal{E}_{a(i \rightarrow j)}=\mathcal{E}_{i, j}^{\ddagger}-\left\langle\mathcal{E}_{i}\right\rangle$ as follows

$$
\begin{aligned}
\ln \left(\frac{n_{i}}{n_{j}}\right)_{e q} & =\mathcal{S}_{i}-\mathcal{S}_{j}+\left(1-\frac{\left\langle\mathcal{E}_{i}\right\rangle}{\left\langle\mathcal{E}_{j}\right\rangle}\right) \frac{\mathcal{E}_{i, j}^{\ddagger}}{\left\langle\mathcal{E}_{i}\right\rangle} \\
& =\mathcal{S}_{i}-\mathcal{S}_{j}+\left(1-\frac{\left\langle\mathcal{E}_{i}\right\rangle}{\left\langle\mathcal{E}_{j}\right\rangle}\right)\left(1+\frac{\mathcal{E}_{a}}{\left\langle\mathcal{E}_{i}\right\rangle}\right)
\end{aligned}
$$

\section{Reconnection of the energetic parameter of rate constants to Boltzmann's energy distribu- tion}

\subsection{Starting from the geometric distri- bution}

One of the main goals of present proposal is to unify kinetic and thermodynamic rules under the same law, which can be derived from a simple discrete treatment. Mix $E$ white balls and $N$ black balls, $E$ and $N$ being positive integers, into a large bag and draw them at random. The number of balls $E$ between two balls $N$, written $\mathcal{E}$, is supposed to correspond to the number of energy quanta in a particle, whose mean number is $\langle\mathcal{E}\rangle=E / N$. Simple probabilities say

$$
P\left(\mathcal{E} \geqslant \mathcal{E}^{\ddagger}\right)=\left(\frac{E}{E+N}\right)^{\mathcal{E}^{\ddagger}}=\left(\frac{\langle\mathcal{E}\rangle}{1+\langle\mathcal{E}\rangle}\right)^{\mathcal{E}^{\ddagger}}
$$

For $\mathcal{E}^{\ddagger}$ and $\langle\mathcal{E}\rangle$ large and of the same order of magnitude, this discrete geometric distribution can be approximated as the continuous exponential distribution.

$$
P\left(\mathcal{E} \geqslant \mathcal{E}^{\ddagger}\right)=\mathrm{e}^{-\frac{\mathcal{E}^{\ddagger}}{\langle\mathcal{E}\rangle}}
$$

If we no longer reason in term of threshold but of exact value, we have

$$
\begin{aligned}
P\left(\mathcal{E}=\mathcal{E}^{\ddagger}\right) & =\left(\frac{E}{E+N}\right)^{\mathcal{E}^{\ddagger}}\left(\frac{N}{E+N}\right) \\
& =\left(\frac{\langle\mathcal{E}\rangle}{1+\langle\mathcal{E}\rangle}\right)^{\mathcal{E}^{\ddagger}} \frac{1}{1+\langle\mathcal{E}\rangle}
\end{aligned}
$$

Switching once again to the continuous distribution, in the thermodynamic limit

$$
\begin{aligned}
P\left(\mathcal{E}=\mathcal{E}^{\ddagger}\right) & \sim \mathrm{e}^{-\frac{\mathcal{E}^{\ddagger}}{\langle\mathcal{E}\rangle}}\left(1-\mathrm{e}^{-\frac{1}{\langle\mathcal{E}\rangle}}\right) \\
& =\frac{\mathrm{e}^{-\mathcal{E}^{\ddagger} /\langle\mathcal{E}\rangle}}{\sum_{j=0}^{\infty} \mathrm{e}^{-j /\langle\mathcal{E}\rangle}}
\end{aligned}
$$

which is, when multiplying the numbers of energy quanta by the energy unit $\mathfrak{q}$ and when $\langle\mathcal{E}\rangle \mathfrak{q}=k_{B} T$, the Boltzmann probability law where the denominator can be understood as the sum of all possible microstates, called the partition function. It was considered by Feynman as the summit of statistical mechanics 5, but is recovered in two lines from the exponential distribution. As a consequence, the relative subpopulations of energies $\mathcal{E}_{1}$ and $\mathcal{E}_{2}$ are given by the ratio of two Eq.(10b)

$$
\frac{P_{\left(\mathcal{E}=\mathcal{E}_{1}\right)}}{P_{\left(\mathcal{E}=\mathcal{E}_{2}\right)}}=\mathrm{e}^{\frac{\mathcal{E}_{2}-\mathcal{E}_{1}}{\langle\mathcal{E}\rangle}}
$$

Note that the approximations used above are based on the following property of the exponential function

$$
\lim _{\varepsilon \rightarrow 0}(1+\varepsilon x)^{1 / \varepsilon}=\mathrm{e}^{x}
$$


which can be written

$$
\lim _{q \rightarrow 1}(1+(1-q) x)^{1 /(1-q)}=\mathrm{e}^{x}
$$

that is the $q$-exponential or generalized exponential function which, when $q$ differs from 1, expands the spectrum of statistical mechanics [6] and has applications in a variety of fields, thermodynamical and nonthermodynamical [7. This function has been proposed as a generalization of Arrhenius law [8] and is used in rate theories [9, 10, 11.

\subsection{Simplified Maxwell-Boltzmann dis- tribution}

When $\langle\mathcal{E}\rangle$ corresponds to $k_{B} T$, with energy units, the structure of Eq.(10b) corresponds exactly to that of the
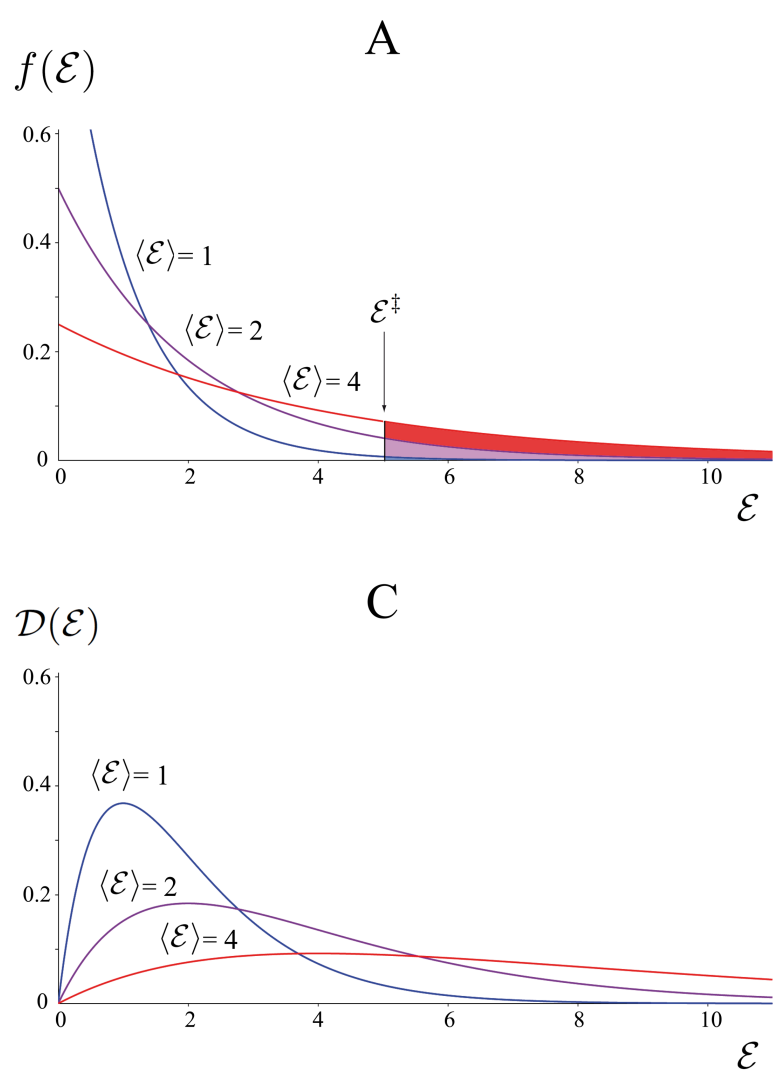

celebrated Boltzmann's energy distribution, valid for example for ideal gases. The ideal monoatomic gas is the perfect example of a system with a maximal entropy but whose individual components have an energy strictly proportional to temperature. Inversely, most real systems have a certain degree of organization (non-maximal entropy) but are made of molecular components with a certain degree of entropy, which will be examined later. Let us first deepen the analogies between the exponential probability distribution and Maxwell-Boltzmann results. The probability density function (PDF) of the exponential distribution of particle energies is

$$
f(\mathcal{E})=\frac{1}{\langle\mathcal{E}\rangle} \mathrm{e}^{-\frac{\mathcal{E}}{\langle\mathcal{E}\rangle}}
$$

represented in Fig.3A.
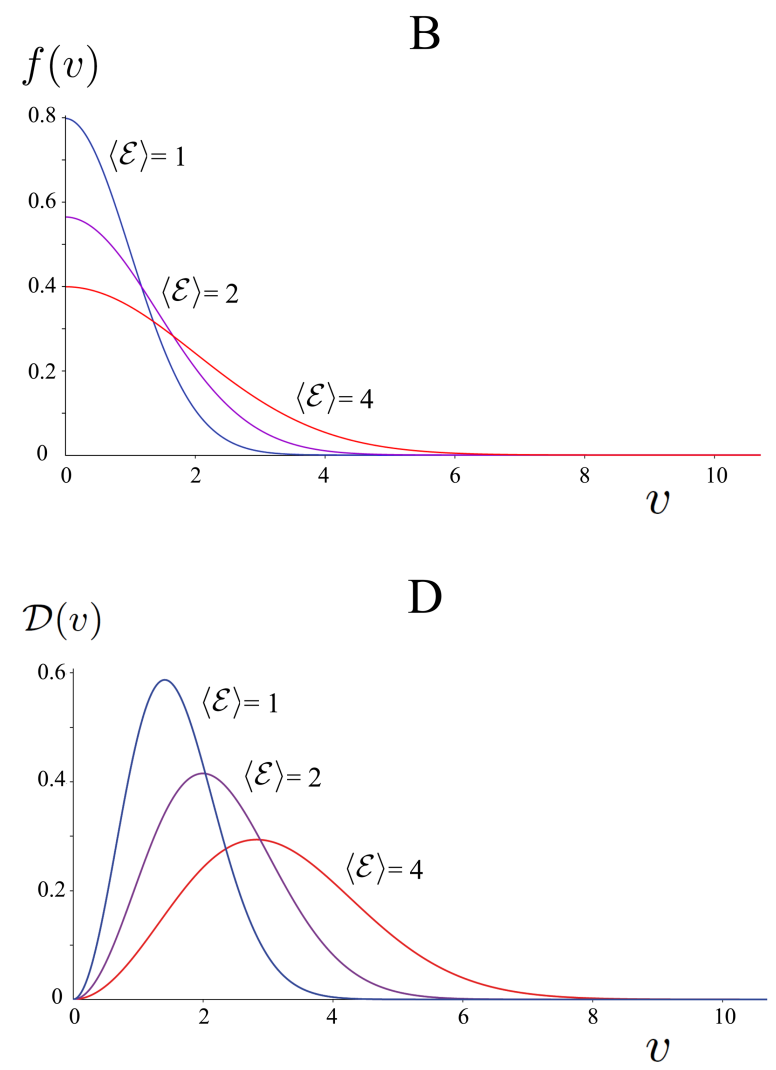

Figure 3. Fractional distributions of: (A) particle energetic states, (B) particle velocities, (C) energy density, and (D) velocity density. Curves drawn with mass and energy of 1 unit. The fractions of particles with energy higher than $\mathcal{E}^{\ddagger}$ correspond to the colored surfaces in panel A, quantified in Eq.(17a). They increase with $\langle\mathcal{E}\rangle$, itself increasing with temperature. This result meets the traditional interpretation of the role of temperature on rate constants, which supposes that particle energies increase with temperature, contrary to Gibbs free energies.

Given that the $n$th moment of this distribution is $E\left[X^{n}\right]=\langle\mathcal{E}\rangle^{n} n$ !, its typical fluctuation is simply

$$
\sigma_{\mathcal{E}}=\sqrt{E\left[X^{2}\right]-E[X]^{2}}=\langle\mathcal{E}\rangle
$$

and a variance of $\langle\mathcal{E}\rangle^{2}$ is acceptable without breaking equilibrium. For a monoatomic ideal gas, the energy content of a particle is exclusively translational. With an energy unit $\mathfrak{q}$ (in joules) such that $\langle\tilde{\mathcal{E}}\rangle=\langle\mathcal{E}\rangle \mathfrak{q}$, a particle of energy $\mathcal{\mathcal { E }}_{i}$ has a velocity $v_{i}$ such that $\tilde{\mathcal{E}}_{i}=\frac{1}{2} m v_{i}^{2}$, giving the distribution of particle velocities, after nor- 
malization,

$$
f(v)=\sqrt{\frac{2 m}{\pi\langle\tilde{\mathcal{E}}\rangle}} \mathrm{e}^{-\frac{m v^{2}}{2\langle\tilde{\mathcal{E}}\rangle}}
$$

represented in Fig.3B and which has a mean value $\bar{v}=\int_{0}^{\infty} v f(v) d v=\sqrt{2\langle\tilde{\mathcal{E}}\rangle / \pi m}$. The particles which have an energy content $\mathcal{E}_{i}$ constitute a fraction of the system $n_{i} / N$ and bring together in the system a fraction of energy $f\left(\mathcal{E}_{i}\right) \mathcal{E}_{i}$. The most represented particles are, according to the exponential as well as Boltzmann distributions, the low-energy and low-mobility particles, which poorly contribute to the total energy of the system, thus generating a peak in the energy density curve at the level of the largest product $\mathcal{E}_{i} f\left(\mathcal{E}_{i}\right)$. The normalized expression of this curve is the density of the $\Gamma_{2}$ function (shape shown in Fig.3C).

$$
\mathcal{D}(\mathcal{E})=\gamma_{2}(\mathcal{E})=\frac{\mathcal{E}}{\langle\mathcal{E}\rangle^{2}} \mathrm{e}^{-\frac{\mathcal{E}}{\langle\mathcal{E}\rangle}}
$$

This function can also be understood as the convolution of the densities of two independent exponential distributions of identical parameter, corresponding to the bottom and top boundaries of the energetic content. For exclusively translational particles, when substituting $\tilde{\mathcal{E}}_{i}=\frac{1}{2} m v_{i}^{2}$,

$$
\mathcal{E}_{i} f\left(\mathcal{E}_{i}\right)=\frac{m v_{i}^{2}}{2\langle\tilde{\mathcal{E}}\rangle} \mathrm{e}^{-\frac{m v_{i}^{2}}{2\langle\tilde{\mathcal{E}}\rangle}}
$$

whose integration between 0 and $\infty$ gives $\sqrt{\pi\langle\tilde{\mathcal{E}}\rangle / 8 m}$. Multiplying the latter equation by the inverse of this value directly yields the normalized velocity density function

$$
\mathcal{D}(v)=\left(\frac{2}{\pi}\right)^{1 / 2}\left(\frac{m}{\langle\tilde{\mathcal{E}}\rangle}\right)^{3 / 2} v^{2} \mathrm{e}^{-\frac{m v^{2}}{2\langle\tilde{\mathcal{E}}\rangle}}
$$

which gives the bell-shaped curves shown in Fig.3D, with a mode at

$$
v_{m p}=\sqrt{\frac{2\langle\tilde{\mathcal{E}}\rangle}{m}}
$$

Therefore, we verify that the most probable values of energy and velocity densities (tips of the curves of Fig.3C and $3 \mathrm{D}$ respectively), naturally coincide through $\tilde{\mathcal{E}}_{m p}=$ $\frac{1}{2} m v_{m p}^{2}$. The mean velocity density $\int_{0}^{\infty} v \mathcal{D}(v) d v$ is

$$
\langle v\rangle=\sqrt{\frac{8\langle\tilde{\mathcal{E}}\rangle}{\pi m}}
$$

When substituting $\langle\tilde{\mathcal{E}}\rangle=k_{B} T$, Eq.(12) is exactly the celebrated Maxwell velocity distribution, currently introduced in different and longer ways. Let us now consider more elaborate systems with different types of particles and energies.

\section{Comparative influences of tem- perature in the different treat- ments}

\subsection{Influence of temperature on rate constants}

If the mean reactional energies are proportional to temperature $(H=C T)$, the traditional shape of the Arrhenius plot is obtained with the present treatment, but through a different way. Increasing the temperature increases the thermal energy stored in the particles and responsible for their reactional capacity, thus reducing the activation energy $E_{a}=E^{\ddagger}-H$. By contrast using the conventional formula of Eq.(1), $E_{a}$ is the constant steering coefficient of the Arrhenius plot [12, in spite of its frequently noticed dependence on temperature [8, 13, and the effect of temperature is assumed to come from the entity $k_{B} T$.

\subsection{Influence of temperature in equilib- rium}

\subsubsection{Where is the temperature in the different equations?}

The specificities of the new proposal can be clearly highlighted by taking up the statistical definition of entropy and grouping together the common elements as follows. The classical relationship can be written

$$
\ln \frac{n_{i}^{e q}}{\Omega_{i}}-\ln \frac{n_{j}^{e q}}{\Omega_{j}}=\frac{H_{j}-H_{i}}{k_{B} T}
$$

while that proposed here is

$$
\ln \frac{n_{i}^{e q}}{\Omega_{i}}-\ln \frac{n_{j}^{e q}}{\Omega_{j}}=E_{i, j}^{\ddagger}\left(\frac{1}{H_{i}}-\frac{1}{H_{j}}\right)
$$

The two major differences between the right sides of Eqs.(13) and (14) are (i) the absence of the energy barrier $E^{\ddagger}$ in the classical form and (ii) the (apparent) absence of the temperature in the new one. It is precisely proposed here that the effect of temperature is mediated by enthalpies, whereas in the classical approach, temperature is also present explicitly in the component $k_{B} T$. Using equilibrium constants for the classical approach, Eq.(13) 
is equivalent to

$$
\ln K_{j i}=\frac{G_{j}-G_{i}}{k_{B} T}
$$

Writing $\Delta G=\Delta H-T \Delta S$ and supposing that $H$ and $S$ are temperature-independent, has the practical advantage to yield the expected van't Hoff plots linearly dependent of $1 / T$. In the present treatment, using an energy threshold $E^{\ddagger}$ and a mean enthalpy $H$ for a reactant or a group of reactants according to the Hess's law,

$$
\ln K_{j i}=\mathcal{S}_{i}-\mathcal{S}_{j}+E^{\ddagger}\left(\frac{1}{H_{i}}-\frac{1}{H_{j}}\right)
$$

showing that when the compounds have the same enthalpy, their equilibrium is governed by entropies. Using the activation energy $E_{a}$,

$$
\ln K_{j i}=\mathcal{S}_{i}-\mathcal{S}_{j}+\left(1-\frac{H_{i}}{H_{j}}\right)\left(1+\frac{E_{a}(i \rightarrow j)}{H_{i}}\right)
$$

Temperature is not apparent in these equations but hidden in the enthalpies. The relative temperaturedependence of the enthalpies regulate the equilibrium, as schematized in Fig.4.
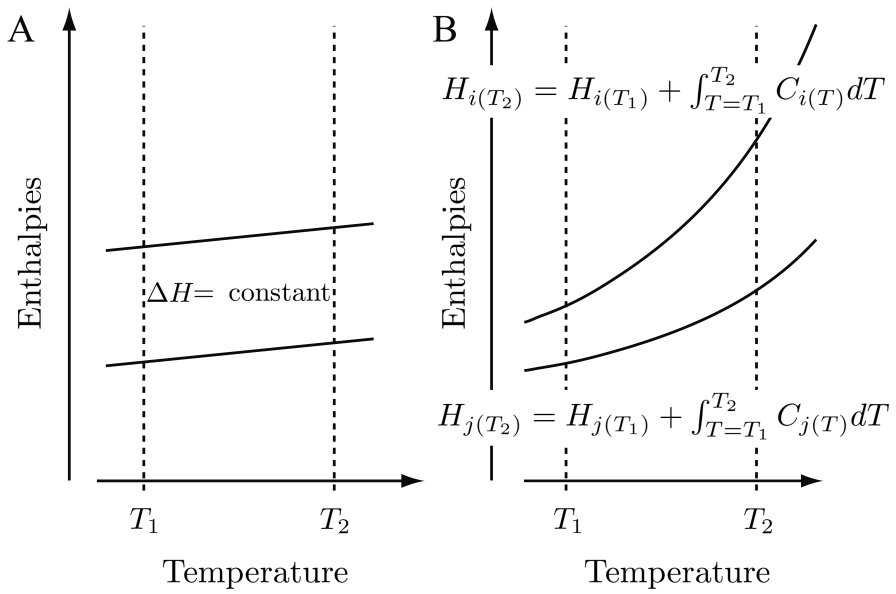

Figure 4. Comparative interpretation of the role of temperature in van't Hoff plots. (A) In the classical interpretation, the heat capacities of the two reactants are always parallel and may vary or not. (B) In the present interpretation, temperature affects in a different manner the reactivity of both reactants because of their different heat capacities. As a consequence, $\Delta H$ depends on temperature. The variations of enthalpies are exaggerated in this scheme, but even a faint divergence between the reactant enthalpies would have exponential consequences on the difference of waiting times between the back and forth reactions.

In the classical interpretation (Fig.4A), the enthalpies of the reactants are shown to increase in parallel with temperature, but note that strangely even if they simply remain constant, the traditional temperaturedependence of van't Hoff plots would still be obtained.

\subsubsection{The kinetic formalism using Gibbs free en- ergies}

Temperature is intuitively expected to increase the reaction rates by increasing the energy of the reactants, but using free energies in the kinetic treatments would say the opposite. $G$ always decreases with temperature, thereby increasing the difference $E^{\ddagger}-G$ and raising the height of the barrier. Using the so-called enthalpies of reaction $H$ instead of Gibbs free energies, allows to circumvent this contradiction. $H$ is indeed proportional to temperature in certain ranges of temperature and does not correspond to $G$. The energy landscape of Arrhenius is governed by enthalpies whereas thermodynamic landscapes include entropies. The activated complex theory describes the kinetic barrier in term of difference of Gibbs free energies [4, 14. In this theory, the tip of the barrier is not a temperature-independent threshold but the energy of an activated complex that itself depends on temperature, so that the temperature-dependence of the height of the barrier $\Delta G^{\ddagger}$ results from the relative dependences on temperature of the ground $(G)$ and transition $\left(G^{\ddagger}\right)$ states [15. The activated complex is an atypical structure stably standing on a saddle point of the energetic landscape and with the strange property, when it is reached, to only evolve in the direction of the complete transition but cannot turn back. Moreover, the forbidden transition is not the same depending of the starting reactant 3. In the kinetic theory, temperature is the macroscopic manifestation of microscopic motions, that is re-scaled at 
the microscopic level through the Boltzmann constant, so that $k_{B} T$ is an average index of single particle motion. But temperature is also present in Gibbs free energy, not only in its most frequent expression $G=H-T S$, but also because $H$ and $S$ are themselves temperature-dependent [16, 17. Raising temperature from $T_{1}$ to $T_{2}\left(>T_{1}\right)$ increases enthalpy by $H\left(T_{2}\right)-H\left(T_{1}\right)=C_{P}\left(T_{2}-T_{1}\right)$ and entropy by $S\left(T_{2}\right)-S\left(T_{1}\right)=C_{P} \ln \left(T_{2} / T_{1}\right)$, where $C_{P}$ is the heat capacity supposed constant at constant pressure.

\subsection{Reintroducing temperature in reac- tional energies}

\subsubsection{Increasing particle energy favors reactions}

Using the energy quanta approach, the dependence on temperature is conferred by the ratio $\mathcal{E}^{\ddagger}$ (temperatureindependent) over $\langle\mathcal{E}\rangle$ (temperature-dependent). This is in fact less clear for the traditional Eq.(15) and the exponent of the rate equation based on a difference of free energy 15

$$
k=\tau^{-1} \mathrm{e}^{-\frac{\Delta G^{\ddagger}}{k_{B} T}}
$$

where both the numerator and the denominator are temperature-dependent. Reactions are facilitated when the energy of the reactants is higher. Possibly because this reasoning does not hold for Gibbs free energies, many teachers prefer drawing the Maxwell velocity distribution to show that increasing the temperature increases the fractional population over the activation energy threshold. This view can in fact be recovered probabilistically, without returning to the ideal gas, using the partial integration of the PDF of the exponential distribution.

$$
\begin{aligned}
P\left(\mathcal{E} \geqslant \mathcal{E}^{\ddagger}\right) & =\int_{\mathcal{E}=\mathcal{E}}^{\infty} \frac{1}{\langle\mathcal{E}\rangle} \mathrm{e}^{-\frac{\mathcal{E}}{\langle\mathcal{E}\rangle}} d \mathcal{E} \\
& =\mathrm{e}^{-\frac{\mathcal{E}^{\ddagger}}{\mathcal{L}\rangle}}
\end{aligned}
$$

One finds again the energetic part of rate constants proposed here, given in Eq.(5) and illustrated by the colored surfaces in Fig.3A. Remarkably, Boltzmann probability can also be obtained by integration of the same function, but in a different manner.

$$
\begin{aligned}
P\left(\mathcal{E}=\mathcal{E}^{\ddagger}\right) & =\int_{\mathcal{E}=\mathcal{E} \ddagger}^{\mathcal{E}^{\ddagger}+1} \frac{1}{\langle\mathcal{E}\rangle} \mathrm{e}^{-\frac{\mathcal{E}}{\langle\mathcal{E}\rangle}} d \mathcal{E} \\
& =\left(1-\mathrm{e}^{-\frac{1}{\langle\mathcal{E}\rangle}}\right) \mathrm{e}^{-\frac{\mathcal{E}^{\ddagger}}{\langle\mathcal{E}\rangle}}
\end{aligned}
$$

The global shift of the Boltzmann distribution upon temperature increase (Fig.3) is commonly used in textbooks as a pedagogical explanation of the increase of the fraction of particles with a energy higher than a given activation threshold. This description implicitly assumes that the Boltzmann distribution of energy is maintained in homogeneous reactants in presence of particle transformations. This rational explanation of the role of temperature mediated by particle energies is formally modeled using Eq.(17a). On the one hand, it can be understood for enthalpies but not for Gibbs free energies, but on the other hand, even when using enthalpies, the current and present approaches strikingly differ, as explained below.

\subsubsection{Temperature increases the reactional en- ergy of the particles}

The dimensionless exponents of the two competing structures of rate constants compared here, contain energies in the numerator and denominator, but beyond this common property, the principles are different. In the usual form, the exponent is $H / k_{B} T$, whereas in the present one, it is $E^{\ddagger} / H$. In the former ratio, the numerator and the denominator both depend on temperature, whereas in the latter one, only the denominator is temperature-dependent. In other words, the traditional formulas still work even when $H$ are independent of temperature, in contradiction with the interpretation of the role of temperature through particle energies. Thermal energy increases the speed of molecules, important for the reactional efficacy of collisions, but more generally the heat capacity of molecules describes how much energy can be stored by a molecule in its internal vibrations or rotations, which are often the triggering factors of chemical transformations, particularly for biological macromolecules whose heat capacity is clearly not negligible. The heat capacity of a monoatomic gas is purely translational, but that of more complex particles include the other forms of energy. In the new exponent proposed here, the denominator represents the temperature-dependent reactional energy in all its forms: translational, rotational and vibrational. Since all these forms define the macroscopic notion of temperature, the reactional propensity is naturally expected to follow temperature. This property precisely characterizes enthalpy, which obeys the thermodynamic relation $\partial H(T) / \partial T=C_{p}$ whose integration with $C_{p}$ temperature-independent and the postulated initial value $H=0$ at $T=0$, simply gives $H \propto C T$. The variation of enthalpies with temperature is in fact less simple in practice. It is calculated using Kirchhoff's law in term of a reference state different from $T=0$ [18. In addition, the temperature-dependence of $C_{P}$ should be introduced using empirical expressions found in tables like $C_{P}=\alpha+\beta T+\gamma T^{-2}+\ldots$ Absolute enthalpies are refractory to experimental measurements contrary to differences of enthalpy calculable by calorimetry. Hence for simplicity in the following equations, the proportionality between $H$ and $T$ will be retained as a gross approxi- 
mation for theoretical elementary reactants. Introducing this definition of enthalpy in Eq.(16) gives

$$
\ln K_{j i} \sim \mathcal{S}_{i}-\mathcal{S}_{j}+\frac{E_{i, j}^{\ddagger}}{T}\left(\frac{1}{C_{i}}-\frac{1}{C_{j}}\right)
$$

Where $C_{i}$ and $C_{j}$ are the average heat capacities of the interconvertible reactants weighted by the stoichiometric coefficients. Strikingly, Eq.(18) can yield typical van't Hoff plots. It also shows that at very high temperature, the equilibrium constant becomes essentially a matter of entropy, as expected. Considering the exponential term of rate constants as a probability implies profound conceptual changes but moderate apparent changes in practice, which just impose to reinterpret the experimental plots, as schematized in Fig.5.
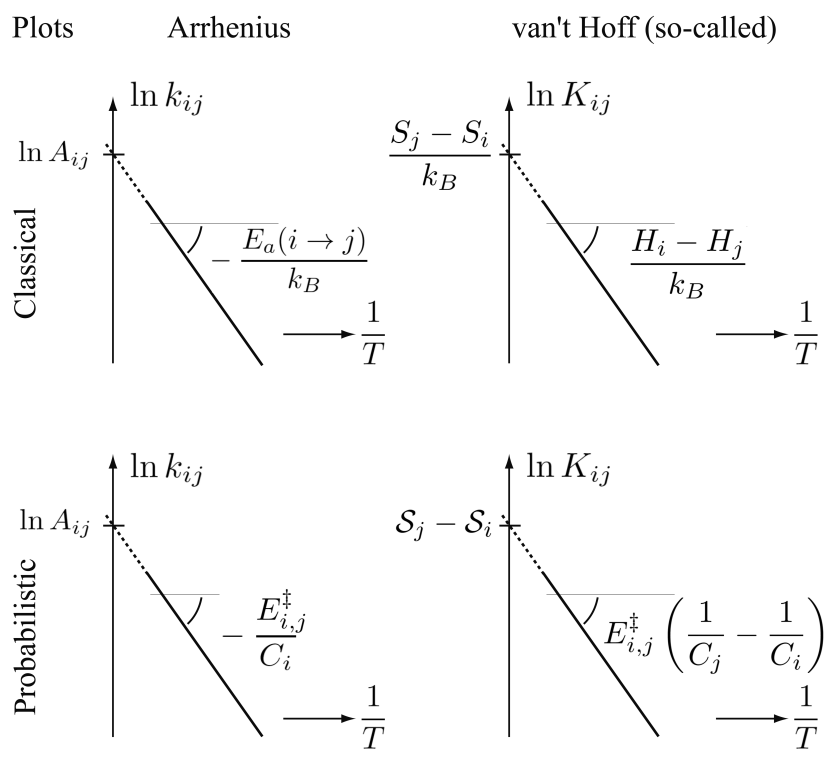

Figure 5. Comparative interpretations of the Arrhenius and van't Hoff plots between the classical and the present treatments of the energetic exponential. The meaning of the entropy intersect is unchanged but that of the slopes is completely different.

The most striking difference is that in the classical interpretation of the van't Hoff plot, the temperaturedependence of enthalpies is denied, whereas the present probabilistic model is fundamentally based on calorimetric enthalpies. The simplistic dependence on temperature depicted in Fig. 5 should be further refined in these very approximate equations, using Kirchhoff's law, setting the standard values $H_{0}$ at $T=298 K$, and taking into account the dependence on temperature of heat capacities, entropies and enthalpies according to the basic thermodynamic differentials

$$
\frac{\partial S(T)}{\partial T}=\frac{C_{p}}{T}
$$

$$
\frac{\partial H(T)}{\partial T}=C_{p}
$$

The frequency of the preexponential factor is also temperature-dependent. Moreover, certain ranges of temperature should not be considered in case of phase transition or of inactivation of certain reactants. For example, proteins are denatured over $320 \mathrm{~K}$ and frozen below $270 \mathrm{~K}$.

\subsubsection{Deviations from the expected plots}

Experimental Arrhenius plots are rarely the straight lines expected from the Arrhenius equation $[\ln k=\ln A-$ $\left.\frac{E_{a}}{R} \frac{1}{T}\right]$, but can have convex or concave shapes, explained by tunneling effects and other hypotheses [8, 19, 20. The dependence of heat capacities on temperature is an additional explanation. Deviations are also the rule for the so-called van't Hoff plots in equilibrium. Moreover, the two types of enthalpy: (i) calorimetric and (ii) determined from the analysis of experimental plots using the currently admitted functions, are always different [16, 17. Reintroducing the temperature-dependence of enthalpies and entropies might account for certain deviations [21, 22, but more fundamentally, a difference of enthalpy between the interconvertible reactants is synonymous to a heat of reaction, that itself reflects a difference of heat capacity, and heat capacities generally vary with temperature.

\subsection{Giving a calorimetric meaning to the Boltzmann constant}

Heat capacity is assumed to be closely related to energy fluctuations. We have seen that the typical fluctuation of the exponential distribution is $\sigma_{\mathcal{E}}=\langle\mathcal{E}\rangle$. Considering it as proportional to temperature through an absolute heat capacity $C$, gives $\sigma_{\mathcal{E}}=C T$, to be compared with the result of statistical mechanics $\sigma_{E}=\sqrt{C_{V} k_{B}} T$ obtained using $\langle E\rangle=U=-\frac{1}{Z}(\partial Z / \partial \beta)_{N, V}, \beta=1 /\left(k_{B} T\right)$ and $C_{V}=(\partial U / \partial T)_{N, V}$. This comparison suggests a correspondence between the Boltzmann constant and a heat capacity. This correspondence is also supported by the proportionality, in the classical limit and following the Dulong-Petit law, between heat capacity and Boltzmann constant in simple systems like ideal gases. In constant volume, the heat capacity of ideal gases is $C_{V}=\frac{n}{2} k_{B}$, where $n$ is the number of degrees of freedom. For instance, ideal monoatomic gas particles flying in a constant volume have a mean kinetic energy

$$
C_{V} T=\frac{3}{2}\langle\tilde{\mathcal{E}}\rangle=\tilde{\mathcal{E}}_{\mathrm{rms}}=\frac{1}{2} m v_{\mathrm{rms}}^{2}
$$

where $v_{\text {rms }}=\sqrt{3\langle\tilde{\mathcal{E}}\rangle / m}$ is the root mean square velocity, obtained using velocity vectors split over the 3 spatial coordinates. The interest of taking $k_{B}$ as a minimalist heat 
capacity is twofold: $k_{B} T$ would be a prototypal enthalpy for simple systems and the two entropies, thermodynamic and statistical, would be related through a heat capacity. Volume changes regulate pressure for systems of mutually colliding particles like gases, but for macromolecules in solution, pressure is essentially due to microscopic collisions with the solvent, water, that is itself incompressible and whose cellular content automatically adjusts to the cellular volume. Heat capacity defines the extent of heat absorbed or released upon temperature change, according to $\Delta Q=C \Delta T$. But at constant temperature if for some reason the heat capacity evolves, then a release or uptake of heat is also expected such that $\Delta Q=T \Delta C$, thus making a clear connection between changes of heat capacity and of thermodynamic entropy, the latter one including in addition the configurational information of statistical entropy such that $S=C \mathcal{S}$. Under this postulate, heat capacities would become the main ingredients of the thermodynamic constants. For the equilibrium constant,

$$
\ln K_{j i}=\left(\frac{S_{i}}{C_{i}}-\frac{S_{j}}{C_{j}}\right)+\frac{E_{i, j}^{\ddagger}}{T}\left(\frac{1}{C_{i}}-\frac{1}{C_{j}}\right)
$$

and for the kinetic constant,

$$
\ln k_{i j}=-\ln \tau-\frac{1}{C_{i}}\left(S_{i}+\frac{E_{i, j}^{\ddagger}}{T}\right)
$$

Differentiation of Eq.(19) with respect to temperature gives

$$
\frac{d \ln K_{j i}}{d T}=\left(C_{i}-C_{j}\right) \frac{E_{i, j}^{\ddagger}}{C_{i} C_{j}} \frac{1}{T^{2}}
$$

which is interesting since Eq.(21) has the structure of the original equation of van't Hoff, based on the heat developed by the reaction 23. Using conventional Boltzmann entropies $S=k_{B} \mathcal{S}$, differentiation of Eq.(18) gives

$$
\frac{d \ln K_{j i}}{d T}=\left(C_{i}-C_{j}\right)\left(\frac{1}{k_{B} T}+\frac{E_{i, j}^{\ddagger}}{C_{i} C_{j} T^{2}}\right)
$$

Once again the theoretical heat capacities of these equations are in fact $H / T$.

\subsection{Extension of the detailed balance to activation energies}

To the properties of the hypothetical rate constants listed above, is added a complication to satisfy the detailed balance rule linking the different rates constants of a system.
The traditional form of the energetic exponential obviously complies with this rule since the total difference of energy of a global reaction made of several elementary steps is simply the sum of energy gaps, with the automatic elimination of activation energies, as illustrated in Eq.(2d). Things are less simple with the new form since the activation energies should now take part to the detailed balance relationship. Consider for example a triangular circuit. The compounds $a$ and $b$ can interconvert either directly, with an activation threshold $E_{a, b}^{\ddagger}$, or indirectly through an intermediate $c$. Assuming that the reactional enthalpy and entropy of every reactant are the same for all its transformations, writing $E_{a, c}^{\ddagger}$ the activation threshold between $a$ and $c$, and $E_{c, b}^{\ddagger}$ between $c$ and $b$, the detailed balance relation

$$
\frac{k_{a b} k_{b c} k_{c a}}{k_{b a} k_{c b} k_{a c}}=1
$$

implies for the energies

$$
\frac{E_{a, c}^{\ddagger}-E_{a, b}^{\ddagger}}{H_{a}}+\frac{E_{a, b}^{\ddagger}-E_{b, c}^{\ddagger}}{H_{b}}+\frac{E_{b, c}^{\ddagger}-E_{a, c}^{\ddagger}}{H_{c}}=0
$$

generalizable for all the cycles of the system, with any number of components $n$

$$
\sum_{i=1}^{n} \frac{E_{i, i-1}^{\ddagger}-E_{i, i+1}^{\ddagger}}{H_{i}}=0 \quad \text { with } \quad E_{1,0}^{\ddagger}=E_{n, n+1}^{\ddagger}=E_{1, n}^{\ddagger}
$$

These relationships with circular permutations are mutually compatible for all the cycles of a reticulated system. In this new treatment, the activation energies are now envisioned as integral components of the system, whereas in the classical theory, they were extraparameters whose values can be arbitrary.

\section{Rebuilding discrete thermody- namics}

Matter was discretized in the visionary theory of Boltzmann and the same was extended to energy by Planck 24]. Since handling energy as a number of quanta facilitates the formulation of basic equations, let us apply again this recipe to the rudiments of thermodynamics and then reintroduce the new kinetic rules proposed above. In this section, the notions of free energy and entropy will apply to the system while the reactants will be supposed purely enthalpic. The concept of system entropy long proved fruitful to begin statistical physics.

\subsection{Index of uncertainty for a population}

Two criteria are sufficient for defining this index equivalent to entropy. It should be: (i) a function of the number 
of possible configurations (microstates) of the system's components and (ii) additive for uncorrelated systems.

(i) The different possible configurations of a macroscopic system (total number written $\Omega$ ), are invisible and definitely not measurable in practice, but the number of these theoretical "snapshots" can be deduced statistically. It reflects the uncertainty of the system, a notion closely related to missing information and disorganization. For example, a book is an organized object in which the page 189 is located between the pages 187 and 191 with a good degree of confidence, but if the pages are torn and thrown down, this probability strongly decreases because the number of possible arrangements of the pages increases.

(ii) The other requirement for an index of disorganization is its additivity for independent systems. This can also be easily understood: For each microstate of a system 1, all the states of a system 2 are possible, so the total number of states of the global system $1+2$ is the product $\Omega_{1} \Omega_{2}$. Obviously the function satisfying

$$
f\left(\Omega_{1} \Omega_{2}\right)=f\left(\Omega_{1}\right)+f\left(\Omega_{2}\right)
$$

is the logarithmic function $(\ln \Omega)$. We will also define a single particle average index $\mathcal{S}=\frac{1}{N} \ln \Omega$. Concretely, the total number of configurations of a system made of $N$ noninteracting components falling into $r$ categories is

$$
\Omega=\frac{N !}{n_{1} ! n_{2} ! \ldots n_{r} !}
$$

Using the Stirling approximation $x ! \sim x^{x} \mathrm{e}^{-x} \sqrt{2 \pi x}$ 25] of which a rough approximation is $x ! \sim x^{x}$,

$$
\Omega \sim \frac{N^{N}}{n_{1}^{n_{1}} n_{2}^{n_{2}} \ldots n_{r}^{n_{r}}}=\left[\left(\frac{n_{1}}{N}\right)^{n_{1}}\left(\frac{n_{2}}{N}\right)^{n_{2}} \ldots\left(\frac{n_{r}}{N}\right)^{n_{r}}\right]^{-1}
$$

so that

$$
\ln \Omega=-\sum_{i=1}^{r} \ln \left(\frac{n_{i}}{N}\right)^{n_{i}}
$$

giving a single particle average index of

$$
\mathcal{S}=-\frac{1}{N} \sum_{i=1}^{r} n_{i} \ln \left(\frac{n_{i}}{N}\right)=-\sum_{i=1}^{r} p_{i} \ln p_{i}
$$

with $p_{i}=n_{i} / N$ and $\sum_{i} n_{i}=N$. With respect to these definitions, the so-called Boltzmann entropy $\left(S_{B}\right)$ and Gibbs/Shannon entropy $\left(S_{G}\right)$ are not equivalent, but related through $S_{G}=S_{B} / N . S_{G} / k_{B}$ corresponds to $\mathcal{S}$ whereas $S_{B} / k_{B}$ corresponds to $N \mathcal{S}$.

But for deriving a pure theory, using an approximation, as acceptable as it can be, is not satisfactory. To bypass the need for the Stirling approximation, it is of some interest to reconsider Eq.(26), which could simply mean that every fractional state $n_{i} / N$ is occupied $n_{i}$ times, so that $\Omega$ is simply the reciprocal of the probability of occupancy. Based on the same principle, another equation can be designed by supposing that the system can evolve.

\subsection{Evolving system}

In a first-order network, $r$ particle states obey a linear evolution system, which has a unique stable solution written $n_{i}^{e q}$. A new state function allows to describe the occupation of the equilibrium proportions $\left(\frac{n_{i}^{e q}}{N}\right)^{n_{i}}$ :

$$
\mathcal{U}=-\frac{1}{N} \sum_{i=1}^{r} n_{i} \ln \left(\frac{n_{i}^{e q}}{N}\right)=-\sum_{i=1}^{r} p_{i} \ln p_{i}^{e q}
$$

$\mathcal{U}$ is an index of the mean gap between the current and equilibrium distributions. This gap can incite the system to evolve, provided it is higher than its degree of disorganization $\mathcal{U}>\mathcal{S}$. The difference $\mathcal{F}=\mathcal{U}-\mathcal{S}$ is

$$
\begin{aligned}
\mathcal{F} & =-\sum_{i=1}^{r} p_{i} \ln p_{i}^{e q}+\sum_{i=1}^{r} p_{i} \ln p_{i} \\
& =\sum_{i=1}^{r} p_{i} \ln \left(\frac{p_{i}}{p_{i}^{e q}}\right)
\end{aligned}
$$

$\mathcal{F}$ will prove particularly interesting upon derivation.

\subsubsection{Introducing time}

To concretize the notion of system evolution and of interchangeability between single particle states, one should introduce a temporal ingredient, in the form of rate constants $k$ examined previously of unit time ${ }^{-1}$. As these rates depend on the temperature, the system will be considered closed but not isolated and embedded in a thermal bath (NVT ensemble). As the different states can be interchanged two by two, a simple two-state system can be used to derive the proportions of particles in states $i$ and $j$ (or for a single particle, the probability to be at a given moment in state $i$ or $j$ ). Let us first derive $\mathcal{S}$.

$$
\begin{aligned}
\frac{d \mathcal{S}}{d t} & =-\left(p_{i} \ln p_{i}+p_{j} \ln p_{j}\right)^{\prime} \\
& =\left(k_{i j} p_{i}-k_{j i} p_{j}\right)\left(1+\ln p_{i}\right) \\
& +\left(k_{j i} p_{j}-k_{i j} p_{i}\right)\left(1+\ln p_{j}\right) \\
& =\left(k_{i j} p_{i}-k_{j i} p_{j}\right) \ln \left(\frac{p_{i}}{p_{j}}\right)
\end{aligned}
$$

which can be generalized to the $r$ nodes

$$
\frac{d \mathcal{S}}{d t}=\sum_{i, j}\left(k_{i j} p_{i}-k_{j i} p_{j}\right) \ln \left(\frac{p_{i}}{p_{j}}\right)
$$


Eq.(31) is not particularly illuminating because it can be either positive or negative; but things are more interesting when deriving $\mathcal{F}$. Taking two interconvertible nodes $i$ and $j$,

$$
\begin{aligned}
\mathcal{F}^{\prime}(i, j)= & {\left[p_{i} \ln \left(\frac{p_{i}}{p_{i}^{e q}}\right)+p_{j} \ln \left(\frac{p_{j}}{p_{j}^{e q}}\right)\right]^{\prime} } \\
& =\left(k_{i j} p_{i}-k_{j i} p_{j}\right)\left[\ln \left(\frac{p_{j}}{p_{j}^{e q}}\right)-\ln \left(\frac{p_{i}}{p_{i}^{e q}}\right)\right] \\
& =-\left(k_{i j} p_{i}-k_{j i} p_{j}\right) \ln \left(\frac{p_{i}}{p_{j}} \frac{p_{j}^{e q}}{p_{i}^{e q}}\right) \\
& \text { and considering that } k_{i j} p_{i}^{e q}=k_{j i} p_{j}^{e q} \\
& =-\left(k_{i j} p_{i}-k_{j i} p_{j}\right) \ln \left(\frac{k_{i j} p_{i}}{k_{j i} p_{j}}\right)
\end{aligned}
$$

which can be written in term of flux $(J=n k)$ for the $r$ nodes,

$$
\frac{d \mathcal{F}}{d t}=-\sum_{i, j}\left(J_{i j}-J_{j i}\right) \ln \left(\frac{J_{i j}}{J_{j i}}\right)
$$

Contrary to Eq.(31), Eq.(32) can be only zero or negative. For this reason, it remarkably illustrates the free energy dissipation and can naturally be identified with the arrow of time [26, 27, 28]. The discrete statistical functions described above can be reconnected to traditional thermodynamics by identifying $k_{B} T \mathcal{F}$ with the Gibbs free energy of the system, $k_{B} T \mathcal{U}$ with the internal energy and $k_{B} \mathcal{S}$ with the entropy. An increase of entropy (towards equipartition) logically decreases free energy and the possibility to extract some work. Further extensions of this discrete approach to thermodynamics are described in [29, 30]. Now let us look at Eq.(32) which has the particularity to bring together the two different aspects of time.

\subsubsection{Time's arrow and time steps}

The arrow of time envisioned as the dissipation of free energy in Eq.(32) has a clear thermodynamic origin out of equilibrium, but even in full equilibrium conditions, the rates constants keep their time units and the particles indefinitely continue to move and transform. This sort of time, completely reversible contrary to the time's arrow, corresponds to the elementary time steps related to another field of physics: quantum physics. Statistical and quantum physics are intimately and necessarily related because enumerating configurations would be infinite in continuous space and time. Accordingly, the quantum of time can be defined as the time necessary to cross the length unit below which successive configurations cannot be distinguished, because of the uncertainty principle. This value can be obtained in multiple ways including in the context of the Maxwell-Boltzmann system. For a single particle, the length unit is the thermal wavelength of de Broglie $\lambda=h / p=h / \sqrt{2 \pi m k_{B} T}$ and the mean particle velocity determined previously is $\bar{v}=\sqrt{2 k_{B} T / \pi m}$. The ratio gives the minimal time step

$$
\tau=\frac{\lambda}{\bar{v}}=\frac{h}{2 k_{B} T} \approx 8 \times 10^{-14} \mathrm{~s} \text { at } T=300 \mathrm{~K}
$$

whose reciprocal is the maximal frequency close to $k_{B} T / h$, considered by Eyring as the most remarkable achievement of his theory 31, and which was already present in the equation of Herzfeld [32]. In the rate constants, this maximal frequency $\tau^{-1}$ is more or less decreased by entropic and energetic restrictions.

The arrow of time is a source of endless debates, particularly interesting when applied to the universe taken as a whole. The free energy of a closed system should necessarily diminish within the limits of residual fluctuations. Then, whence comes the initial free energy of our universe which does not seem very organized in its youth? Did previously latent forces release free energy during the physico-chemical maturation of the universe? or do we live in a local fluctuation in a giant universe in equilibrium? according to an elegant but contested proposal of Boltzmann 33 .

\subsection{Introducing discrete energy}

The frequencies $k_{i j}$ introduced in Section 6.2.1 remain abstract and their nature should be precised. In addition to their temporal parameter, the rate constants include an energy parameter which can be different in the different $k$. Before envisioning reactional systems, the spreading of energy will be first described in simple systems without chemical transformations. Instead of distributing particles into "energy levels", energy units are distributed over particles. Things appear clearer when energy is handled discretely. Perhaps influenced by what Boltzmann said him in 1891: "I see no reason why energy shouldn't also be regarded as divided atomically", Planck proposed in the founder article which opened the way to quantum physics 24], to discretize energy into quanta of value $h \nu$, written more generally $\mathfrak{q}$ below.

\subsubsection{Homogeneous system}

The mean energy of thermal systems can be recovered in a discrete manner from the second law. Returning to the white balls supposed to correspond to energy quanta in Section.4, the number of ways to distribute $E$ objects in $N$ boxes is

$$
\Omega=\frac{(N+E-1) !}{(N-1) ! E !}
$$


which gives, using the Stirling approximation, a single particle average entropy of

$$
\mathcal{S}=\frac{1}{N} \ln \Omega=\left(1+\frac{E}{N}\right) \ln \left(1+\frac{E}{N}\right)-\frac{E}{N} \ln \left(\frac{E}{N}\right)
$$

When the system contains a single category of particles, the ratio $E / N$ is equivalent to the mean number of energy quanta per particle $U / \mathfrak{q}[24$.

$$
\mathcal{S}=\frac{S}{k_{B}}=\left(1+\frac{U}{\mathfrak{q}}\right) \ln \left(1+\frac{U}{\mathfrak{q}}\right)-\frac{U}{\mathfrak{q}} \ln \left(\frac{U}{\mathfrak{q}}\right)
$$

Temperature can now be introduced using the fundamental entropy equation.

$$
\frac{d S}{d U}=\frac{1}{T}
$$

according to which integration of Eq.(35b) yields

$$
\frac{k_{B}}{\mathfrak{q}} \ln \left(1+\frac{\mathfrak{q}}{U}\right)=\frac{1}{T}
$$

and

$$
\frac{U}{\mathfrak{q}}=\langle\mathcal{E}\rangle=\frac{1}{\mathrm{e}^{\mathfrak{q} / k_{B} T}-1}
$$

The reasoning can be circular in statistical thermodynamics and these results can also be recovered in a reverse way starting from the partition function, as did Einstein [34]. The ratio of Planck $(\mathfrak{q}=h \nu)$ over Boltzmann entities, turns to be the logarithm of the inverse proportion of energy quanta in the mixture.

$$
\frac{h \nu}{k_{B} T}=-\ln \frac{E}{N+E}=\ln \left(1+\frac{1}{\langle\mathcal{E}\rangle}\right)
$$

Replacing enthalpy by this energy yields the new Arrhenius esquation

$$
\ln k / A=-\frac{E^{\ddagger}}{\mathfrak{q}}\left(\mathrm{e}^{\mathfrak{q} / k_{B} T}-1\right)
$$

At the thermal scale, $\mathfrak{q}$ is negligible compared to $k_{B} T$ and the expansion of Eq.(37b) gives a mean number (unitless) of energy quanta

$$
\langle\mathcal{E}\rangle \sim \frac{k_{B} T}{\mathfrak{q}}
$$

and a mean energy (joules) of

$$
\langle\mathcal{E}\rangle \mathfrak{q}=U \sim k_{B} T
$$

As the heat capacity of a substance is its ability to increase its energy content with temperature, it can therefore be deduced from Eq.(37b),

$$
C=\mathfrak{q} \frac{d\langle\mathcal{E}\rangle}{d T}=\frac{\mathfrak{q}^{2}}{k_{B} T^{2}} \frac{\mathrm{e}^{\mathfrak{q} / k_{B} T}}{\left(\mathrm{e}^{\mathfrak{q} / k_{B} T}-1\right)^{2}}
$$

\subsubsection{Heterogeneous system}

When the system is composite and made of $r$ different categories of particles which have different mean energies $\left\langle\mathcal{E}_{i}\right\rangle$, we have to fill $r$ boxes with $N=\sum_{i} n_{i}$ particles and $E=\sum_{i} n_{i}\left\langle\mathcal{E}_{i}\right\rangle$ energy quanta

$$
\Omega=\prod_{i=1}^{r} \frac{\left(n_{i}+E_{i}-1\right) !}{\left(n_{i}-1\right) ! E_{i} !}
$$

giving, for large populations and using the Stirling approximation, an entropy of

$$
\ln \Omega=\sum_{i=1}^{r} n_{i}\left[\left(1+\frac{E_{i}}{n_{i}}\right) \ln \left(1+\frac{E_{i}}{n_{i}}\right)-\frac{E_{i}}{n_{i}} \ln \left(\frac{E_{i}}{n_{i}}\right)\right]
$$

Keeping $N$ and $E$ constant, this entropy is lower than that of an homogeneous system, showing that the categorization of objects is a first step towards organization. As the derivative of a sum is the sum of the derivatives,

$$
\left\langle\mathcal{E}_{i}\right\rangle=\frac{E_{i}}{n_{i}}=\frac{1}{\mathrm{e}^{\mathfrak{q} i / k_{B} T}-1}
$$

\section{First-order particle interconversion}

To render this heterogeneous system dynamic, one should introduce the possibility of interconversion between the particles of the different kinds in the form of rate constants. The rule for particle interconversion has been established in Eq.(8) using the exponential law. For unimolecular transconversions which do not modify the total number of particles, let us abandon the ratios of preexponential factors. The single particle energy averaged over the $r(r-1) / 2$ couples of categories in the system is

$$
\left\langle\mathcal{E}_{m}\right\rangle=\frac{E}{N}=\frac{\sum_{i} \sum_{j \neq i}\left\langle\mathcal{E}_{i}\right\rangle \mathrm{e}^{-\frac{\mathcal{E}_{i, j}^{\ddagger}}{\left\langle\mathcal{E}_{i}\right\rangle}}}{\sum_{i} \sum_{j \neq i} \mathrm{e}^{-\frac{\mathcal{E}_{i, j}^{\ddagger}}{\left\langle\mathcal{E}_{i}\right\rangle}}}
$$

in which the inexisting interchanges are characterized by $\mathcal{E}_{i, j}^{\ddagger}=\infty$. For a system of enthalpic components, one can define the transition rate constants

$$
\begin{aligned}
& k_{i j}=\tau^{-1} \mathrm{e}^{-\mathcal{E}_{i, j}^{\ddagger} /\left\langle\mathcal{E}_{i}\right\rangle} \\
& k_{j i}=\tau^{-1} \mathrm{e}^{-\mathcal{E}_{i, j}^{\ddagger} /\left\langle\mathcal{E}_{j}\right\rangle}
\end{aligned}
$$


where $\tau$ is the time step and $\left\langle\mathcal{E}_{i}\right\rangle$ is the mean energy of the particles of type $i$, both depending on temperature through Eq.(33) and Eq.(42) respectively.

For two categories $n_{1}$ and $n_{2}$, the relationships linking energy and particle numbers are

$$
\begin{gathered}
\frac{n_{1}}{N}=\left(1+\mathrm{e}^{\mathcal{E}_{1,2}^{\ddagger}\left(\frac{1}{\left\langle\mathcal{E}_{1}\right\rangle}-\frac{1}{\left\langle\mathcal{E}_{2}\right\rangle}\right)}\right)^{-1} \\
\frac{n_{2}}{N}=\left(1+\mathrm{e}^{\mathcal{E}_{1,2}^{\ddagger}\left(\frac{1}{\left\langle\mathcal{E}_{2}\right\rangle}-\frac{1}{\left\langle\mathcal{E}_{1}\right\rangle}\right)}\right)^{-1} \\
\frac{E_{1}}{E_{2}}=\frac{\left\langle\mathcal{E}_{1}\right\rangle}{\left\langle\mathcal{E}_{2}\right\rangle} \mathrm{e}^{\mathcal{E}_{1,2}^{\ddagger}\left(\frac{1}{\left\langle\mathcal{E}_{1}\right\rangle}-\frac{1}{\left\langle\mathcal{E}_{2}\right\rangle}\right)}
\end{gathered}
$$

and the mean particle energy in the system is

$$
\left\langle\mathcal{E}_{m}\right\rangle=\frac{\left\langle\mathcal{E}_{1}\right\rangle \mathrm{e}^{-\frac{\mathcal{E}_{1,2}^{\ddagger}}{\left\langle\mathcal{E}_{1}\right\rangle}}+\left\langle\mathcal{E}_{2}\right\rangle \mathrm{e}^{-\frac{\mathcal{E}_{1,2}^{\ddagger}}{\left.\mathcal{E}_{2}\right\rangle}}}{\mathrm{e}^{-\frac{\mathcal{E}_{1,2}^{\ddagger}}{\left\langle\mathcal{E}_{1}\right\rangle}}+\mathrm{e}^{-\frac{\mathcal{E}_{1,2}^{\ddagger}}{\left\langle\mathcal{E}_{2}\right\rangle}}}
$$

\section{Circular equilibria}

Three categories of particles are sufficient to establish the famous detailed balance relationship of Wegscheider linking the constants of a cycle [35. The relation

$$
\frac{n_{1}}{n_{2}} \frac{n_{2}}{n_{3}} \frac{n_{3}}{n_{1}}=1
$$

is obviously always true, in particular at equilibrium, so the constants are necessarily related through

$$
\frac{k_{12}}{k_{21}} \frac{k_{23}}{k_{32}} \frac{k_{31}}{k_{13}}=1
$$

The activation energies, which were completely arbitrary in previous theories, are now mutually connected through

$$
\frac{\mathcal{E}_{1,3}^{\ddagger}-\mathcal{E}_{1,2}^{\ddagger}}{\left\langle\mathcal{E}_{1}\right\rangle}+\frac{\mathcal{E}_{1,2}^{\ddagger}-\mathcal{E}_{2,3}^{\ddagger}}{\left\langle\mathcal{E}_{2}\right\rangle}+\frac{\mathcal{E}_{2,3}^{\ddagger}-\mathcal{E}_{1,3}^{\ddagger}}{\left\langle\mathcal{E}_{3}\right\rangle}=0
$$

Circular equilibria are particularly relevant with respect to catalysts. Contrary to the widespread representation of catalysis as a deformation of the energy landscape along the coordinates of a single transition, it should rather be envisioned as a circuit, at least triangular and letting unchanged the uncatalysed single step reaction.

\subsection{Possible source of confusion for mod- eling the relationships between frac- tional populations and energy}

The enthalpic particles of the toy systems described above are sufficient to visualize the important differences in the description of the ratios of particles, depending on whether they belong to the same category or not. For a homogeneous system of mean energy $\left\langle\mathcal{E}_{1}\right\rangle$ in which energy diffuses without barriers between particles $n_{1}$, the ratio of particles with energies $\mathcal{E}_{a}$ and $\mathcal{E}_{b}$ follows, according to $\mathrm{Eq}(10 \mathrm{c})$,

$$
n_{1}(a) / n_{1}(b)=\mathrm{e}^{\frac{\mathcal{E}_{a}-\mathcal{E}_{b}}{\left\langle\mathcal{E}_{1}\right\rangle}}
$$

which is a familiar Boltzmann relation when setting $\left\langle\mathcal{E}_{1}\right\rangle=k_{B} T$, whereas interchangeable particles of different categories with different mean energies $\left\langle\mathcal{E}_{1}\right\rangle$ and $\left\langle\mathcal{E}_{2}\right\rangle$, are related through

$$
n_{1} / n_{2}=\mathrm{e}^{\mathcal{E}_{1,2}^{\ddagger}\left(\frac{1}{\left\langle\mathcal{E}_{1}\right\rangle}-\frac{1}{\left\langle\mathcal{E}_{2}\right\rangle}\right)}
$$

Eq.(48) is valid for a single phase in the pioneer statistical theories, applicable for instance to energy spreading between gas particles, but the object of this manuscript is precisely to propose that this form cannot be extended to chemical reactions. The next tasks in this direction will be to implement these rules from first order to second order networks, to introduce particle entropies, to extend results to entropic open systems in steady state like biochemical systems, for modeling the fundamental ingredients of life: nonlinearity, retroactions and multistability.

\section{Conclusions}

The present probabilistic treatment based on the exponential distribution does not invalidate the MaxwellBoltzmann distribution, but encompasses it as a particular case of homogeneous system with temperaturedependent particle energies. Hence, there is no reason to not extend this approach to the energy-dependence of particle conversions, because rate constants are integral constituents of dynamic systems. The central and original assumption of statistical physics was the modeling of randomness, but randomness is precisely described in the most fundamental way by the exponential law. In fact, the equations of Arrhenius and Boltzmann are two facets of the same law of randomness, unified in the present theory. This is perfectly illustrated by Eqs.(17a) and Eq.(17b) respectively: the first one is the probability that $\mathcal{E} \geqslant \mathcal{E}^{\ddagger}$ and the second one that $\mathcal{E}=\mathcal{E}^{\ddagger}$. To further enlighten this point, Boltzmann probability is simply obtained using the exponential distribution only, by 
subtraction as follows

$$
\begin{aligned}
P(\mathcal{E}=j) & =P(\mathcal{E} \geqslant j)-P(\mathcal{E} \geqslant j+1) \\
& =\mathrm{e}^{-j /\langle\mathcal{E}\rangle}-\mathrm{e}^{-(j+1) /\langle\mathcal{E}\rangle} \\
& =\mathrm{e}^{-j /\langle\mathcal{E}\rangle}\left(1-\mathrm{e}^{-1 /\langle\mathcal{E}\rangle}\right) \\
& =\frac{\mathrm{e}^{-j /\langle\mathcal{E}\rangle}}{\sum_{n=0}^{\infty} \mathrm{e}^{-n /\langle\mathcal{E}\rangle}}
\end{aligned}
$$

The relationships established here are simply derived from the single postulate that the exponential term of rate constants has the status of a probability, in line with the profoundly probabilistic spirit of Boltzmann's theory and with the intuition of Maxwell: "The true logic of this world is in the calculus of probabilities". In this respect, the rate constant defined here is entirely probabilistic

$$
k \tau=P(\text { configuration is } \mathrm{OK}) \times P(\text { energy is } \mathrm{OK})
$$

which simply says that the frequency of a transformation follows the probability of favorable conjunctions of configurational and energetic conditions, which is

$$
k \tau=\frac{1}{\Omega} \times \mathrm{e}^{-\mathcal{E}^{\ddagger} /\langle\mathcal{E}\rangle}
$$

leading to the equilibrium relationship

$$
\ln \frac{n_{i}^{e q}}{\Omega_{i}}-\ln \frac{n_{j}^{e q}}{\Omega_{j}}=\mathcal{E}_{i, j}^{\ddagger}\left(\frac{1}{\left\langle\mathcal{E}_{i}\right\rangle}-\frac{1}{\left\langle\mathcal{E}_{j}\right\rangle}\right)
$$

The mean reactional energies $\langle\mathcal{E}\rangle$ can logically be identified with enthalpies, but Gibbs free energies are not appropriate for these definitions because the entropic and energetic parts are involved in independent probabilities. If the starting probabilistic postulate is wrong, the present study would remain a theoretical exercise, but it is however appealing owing to: (i) its mathematical relevance; (ii) its capacity to recover the Maxwell-Boltzmann distribution and to unify kinetics and thermodynamics; (iii) the recovery of the van't Hoff relation between equilibrium constants and temperature and (iv) the restoration of the link between the reactional propensity and the heat capacity, which is strangely ignored in the traditional interpretation of the slope of van't Hoff plots as $\Delta H / k_{b}$. In the traditional formulas, the enthalpies are not responsible for the dependence on temperature that is due only to the division of enthalpies by $k_{B} T$. By contrast, it is proposed here that enthalpies directly mediate this dependence. Singularly, this view is found in textbooks explaining the dependence of reactions on temperature using populational energy distributions, but without realizing that this explanation contradicts the general formula where the particle energies are at the numerator of the exponent supposed constant, while temperature is at the denominator. But although profoundly restructured, the thermodynamic relationships derived here yield resembling behaviors, so that the comparison between the previous and new treatments is more a question of interpretation of the experimental plots than a true discrimination. In the field of rate theories, Laidler already noticed that, surprisingly, the widely different rate equations proposed in the past can give reasonably good fit to the same experimental data [2. Precise experimental validations in this field are hindered by numerous problems, including the knowledge of absolute enthalpies, the complex temperature-dependence of heat capacities, the interferences with tunnel effects and the narrow range of testable temperature. Moreover, experimental and calculated values are rarely in good agreement [18 and strikingly, calorimetric and van't Hoff enthalpies never coincide [16, 17, which is not a surprise considering that the enthalpy change for a reaction is responsible for a heat of reaction, whereas the heat capacity of enthalpies is ignored in the current van't Hoff plot approach. In the alternative view examined here, the entropies of the reactants are completely identical to the previous ones; the calorimetric enthalpies and the Hess's law are unchanged; the favored direction of reactions is still driven by entropy increase and/or enthalpy decrease; the equilibrium constants remain defined by the ratios of interconvertible reactants concentrations in equilibrium and still satisfies the van't Hoff formula. Note in this respect that the original formula of van't Hoff was not the equation currently attributed to van't Hoff in texbooks, since van't Hoff did not use the Boltzmann's constant or the ideal gas constant, and his reasoning was fundamentally calorimetric 23, in line with the changes of heat capacity on which the present theory is based. In addition, van't Hoff indicated that he managed his equation for numerical applications and experimental controls only, but did not enter into the details of thermodynamics necessary for its demonstration 23. The 19th century formula of Arrhenius also remains an equation of practical convenience, even if experimental data show that it is a poor quantitative predictor of reaction rates, particularly in high-dimensional, complex chemical kinetics. Attempts to rectify this form include transition state theory [4, diffusion from an energy well 36, reactive flux theory 37, and transition path theory [38. The alternative approach proposed here is basically simpler since it is just the probability for a particle in a population to have a given level of energy. The energy barrier is conceived here as a threshold in the Boltzmann distribution of the reactant itself, but not as the energy of a hypothetical complex. The present study, however, deals only with the basic rate constants of unitary transitions between uniform reactants, in which time and energy are clearly separated and the energy barrier is unique. These elementary constants can then be used as ingredients for real reactions which are most often composite and involve non-uniform reactants. (i) Serial reactions can be treated as Markovian walks with absorbing boundaries 
39 in which the probabilities of jumps are transposed to reaction chains by introducing elementary transition rates (unidentifiable in practice), each one with its own energy barrier. The resulting mean first passage times then correspond to the reciprocal of the global reaction rates. Such serial reactions could ensure valuable roles in biochemical interactions [40] and in their accuracy [41. (ii) In biochemistry, real reactants are rarely uniform, particularly in the case of large macromolecules like for example proteins. A protein is a typical example of a giant macromolecule with many chemical bonds and degrees of freedom, clearly subject to so-called dynamic disorder, particularly important in intrinsically disordered domains [40, in which a myriad of microreactions is involved even in simple conformational changes. A protein envisioned as a single species, generally corresponds to an ensemble of conformers with slightly different energies.

The elucidation of the fundamental nature of rate constants is essential considering their widespread use from chemistry to systems modeling. Rate constants are the regulators of systems dynamics and as such allow to introduce the notion of time. If time proves so difficult to conceive, it is probably because it relies on two completely different pedestals belonging to different branches of physics: (i) the step of time, always at work, both out of and in equilibrium and originating from quantum physics and (ii) time arrow, existing only out of equilibrium and which is a populational phenomenon emerging from statistical physics 33. Dynamic systems clarify this perception because they combine both aspects: the time step included in the preexponential component of the Arrhenius equation, and the time arrow describing the spontaneous evolution of a system of interconvertible particle states.

\section{References}

[1] Arrhenius S., Ober die reacktionsgeschwindigkeit bei der inversion von rohrzucker durch sauren, Z. Physik. Chem. 4 (1889) 226-248.

[2] Laidler K.J., The development of the Arrhenius equation, J. Chem. Educ. 61 (1984) 494-498.

[3] Michel D., Simply conceiving the Arrhenius law and absolute kinetic constants using the geometric distribution, Phys. A Stat. Mech. Appl. 392 (2013) 4258-4264. link

[4] Eyring H., The activated complex in chemical reactions, J. Chem. Phys. 3 (1935) 107-115.

[5] Feynman R., Statistical Mechanics Avalon Publishing, 1998.

[6] Tsallis C., What are the numbers that experiments provide, Quimica Nova 17 (1994) 468-471.
[7] Tsallis C., Introduction to nonextensive statistical mechanics: Approaching a complex world. Springer Science \& Business Media 2009.

[8] Aquilanti V., Mundim K.C., Elango M., et al., Temperature dependence of chemical and biophysical rate processes: Phenomenological approach to deviations from Arrhenius law, Chem. Phys. Lett. 498 (2010) 209-213.

[9] Quapp W., Zech A., Transition state theory with Tsallis statistics, J. Comput. Chem. 31 (2010) 573-585.

[10] Yin C., Du J., The power-law reaction rate coefficient for an elementary bimolecular reaction, Phys. A. Stat. Mech. Appl. 395 (2014) 416-424.

[11] Aquilanti V., Coutinho N.D., Carvalho-Silva V.H., Kinetics of low-temperature transitions and a reaction rate theory from non-equilibrium distributions. Philos. Trans. A Math. Phys. Eng. Sci. 375 (2017) pii: 20160201.

[12] Monk P.M.S., Physical Chemistry: Understanding our Chemical World, Wiley, 2004.

[13] Bao J.L., Meana-Pañeda R., Truhlar D.G., Multi-path variational transition state theory for chiral molecules: the site-dependent kinetics for abstraction of hydrogen from 2-butanol by hydroperoxyl radical, analysis of hydrogen bonding in the transition state, and dramatic temperature dependence of the activation energy, Chem. Sci. 6 (2015) 5866-5881.

[14] Evans M.G., Polanyi M., Some applications of the transition state method to the calculation of reaction velocities, especially in solution, Trans. Faraday Soc. 31 (1935) 875894.

[15] Steinfeld J.I., Francisco J.S., Hase W.L., Chemical kinetics and dynamics. Englewood Cliffs, Prentice Hall, NJ. 1989.

[16] Weber G., van't Hoff revisited: Enthalpy of association of protein subunits, J. Phys. Chem. 99 (1995) 1052-1059.

[17] Liu Y., Sturtevant J.M., Significant discrepancies between van't Hoff and calorimetric enthalpies, Prot. Sci. 4 (1995) 2259-2561.

[18] Atkins P.W., de Paula J., Atkins' Physical Chemistry. Oxford University Press, 2010.

[19] Smith I.W.M., The temperature-dependence of elementary reaction rates: beyond Arrhenius, Chem. Soc. Rev. 37 (2008) 812-826.

[20] Masgrau L., González-Lafont A., Lluch J.M., The curvature of the Arrhenius plots predicted by conventional canonical transition-state theory in the absence of tunneling, J. Theor. Chem. Acc. 110 (2003) 352-357.

[21] Naghibi H., Tamura A., Sturtevant J.M., Significant discrepancies between van't Hoff and calorimetric enthalpies, Proc. Natl. Acad. Sci. USA. 92 (1995) 5597-5599.

[22] Sharma G., First E.A., Thermodynamic analysis reveals a temperature-dependent change in the catalytic mechanism of Bacillus stearothermophilus Tyrosyl-tRNA Synthetase, J. Biol. Chem. 284 (2009) 4179-4190. 
[23] van't Hoff J.H., Etudes de Dynamique Chimique (Studies in Chemical Dynamics), F. Muller \& Co, Amsterdam, 1884. link

[24] Planck M., On the law of distribution of energy in the normal spectrum, Ann. Phys. 4 (1901) 553-563.

[25] Michel R., On Stirling's formula, Am. Math. Month. 109 (2002) 388-390.

[26] Luo J.L., van den Broeck C., Nicolis G., Stability criteria and fluctuations around nonequilibrium states, Z. Phys. B Cond. Mat. 52 (1984) 156-170.

[27] Mackey M.C., Time's arrow: The origins of thermodynamic behavior, Springer-Verlag, New York (1992)

[28] Maes C., Netocný K., Time-Reversal and Entropy, J. Stat. Phys. 110 (2003) 269-309

[29] Schnakenberg J., Network theory of microscopic and macroscopic behavior of master equation systems, Rev. Mod. Phys. 48 (1976) 571-585.

[30] Ge H., Qian H., Physical origins of entropy production, free energy dissipation, and their mathematical representations, Phys. Rev. E 81 (2010) 051133.

[31] Glasstone S., Laidler K.J., Eyring H., The theory of rate processes, McGraw-Hill, New York, 1941.

[32] Herzfeld K.F., Zur Theorie der Reaktionsgeschwindigkeiten in Gasen, Ann. Phys. 59 (1919) 635-667.
[33] Lebowitz J.L., Macroscopic laws, microscopic dynamics, time's arrow and Boltzmann's entropy, Physica A. 194 (1993) 1-27.

[34] Einstein A., On the development of our views concerning the nature and constitution of radiation, Phys. Z. 10 (1909) 817-826.

[35] Wegscheider R., Über simultane Gleichgewichte und die Beziehungen zwischen Thermodynamik und Reactionskinetik homogener Systeme, Monatshefte für Chemie/Chemical Monthly 32 (1901) 849-906.

[36] Kramers H.A., Brownian motion in a field of force and the diffusion model of chemical reactions, Physica 7 (1940) 284-304.

[37] Chandler D., Barrier crossings: Classical theory of rare but important events from classical and quantum dynamics in condensed phase simulations. B.J. Berne, G. Ciccotti, D.F. Coker Editors, 1998.

[38] Vanden-Eijnden E., Transition path theory, Lect. Notes Phys. 703 (2006) 439-478.

[39] van Kampen N.G., Stochastic Processes in Physics and Chemistry, 3th Edition, North-Holland Personal Library, Amsterdam, 2003;

[40] Uversky V.N., The multifaceted roles of intrinsic disorder in protein complexes, FEBS Lett. 589 (2015) 2498-2506.

[41] Michel D., Boutin B., Ruelle P., The accuracy of biochemical interactions is ensured by endothermic stepwise kinetics, Prog. Biophys. Mol. Biol. 121 (2016) 35-44. 